\title{
Scheduling Electric Vehicle Charging at Park-and-Ride Facilities to Flatten Duck Curves
}

\author{
Raka Jovanovic \\ Qatar Environment and Energy Research Institute \\ Hamad bin Khalifa University \\ PO Box 5825, Doha, Qatar \\ rjovanovic@hbku.edu.qa
}

\author{
I. Safak Bayram \\ Department of Electrical and Electronic Engineering \\ University of Strathclyde \\ Glasgow, UK \\ safak.bayram@strath.ac.uk
}

\begin{abstract}
In this paper, we explore present a scheduling framework for large-scale electric vehicle charging to flatten duck curves stemming from the imbalance between peak electricity demand and renewable energy production. This situation adds new constraints to power system operations and increases maintenance costs. The focus is on charging systems installed at parkand-ride facilities which are gaining popularity in metropolitan cities. The scheduling problem is modeled as an integer linear problem and various case studies are generated and solved using real-world collected data. The computational experiments show that significant savings can be achieved in reducing power system ramping requirements.
\end{abstract}

\section{INTRODUCTION}

In recent years, there has been an increasing rate of electric vehicle (EV) adoption due to economic and environmental benefits, and the share of electric transportation is expected to rise even further in the near future. In parallel, proliferation of renewable energy sources, especially from renewable solar ones, is changing the electricity utility landscape. It is well known that a high level of solar generation results in the issue known as "duck curve" which requires additional power ramping capabilities due to imbalance between peak demand and renewable energy production [1], [2] (depicted in Fig. 1). Frequent variations of the power generators output accelerates mechanical aging, increase system maintenance cost, and lead to negative electricity prices. Hence, it is desirable by grid operators to improve system reliability and flexibility with demand-side activities and minimize ramping requirements. In this work, we develop a scheduling framework to optimally charge stationary EVs located at park-and-ride facilities to minimize overall ramping requirements.

The scheduling of electrical appliances at residential units is a standard method for lessening the mentioned problem and is a part of many demand response programs [3]. In such approach, the jobs related to home appliances (typically has less than $2 \mathrm{~kW}$ rating) are scheduled to minimize electricity bills. A similar approach can be used in the context of EV charging with Level 2 chargers which are typically deployed at parking lots [4], [5]. In recent years, there has been extensive research on convenient away-from-home charging infrastructure with a special focus on ones located at the workplace [6][8]. In the work of Tulpule et al. [9] the potential benefits of having such systems partially powered using solar energy are

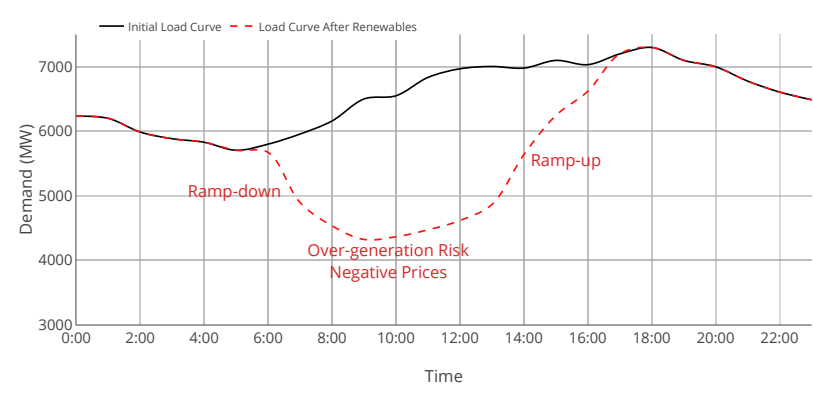

Fig. 1. A typical duck curve.

analyzed. Some interesting concepts and the evaluation of the potential positive effects of smart scheduling of EVs charging at workplace parking lots can be found in references [10], [11] and [12]. Scheduling of stationary EVs present a great opportunity for grid operators to improve system flexibility because EVs are represented by large loads (e.g., 3-7 kW charging for 4-8 hours) compared to residential appliances and charging service can be partially completed as long as a minimum charge level is met.

In this paper, we propose a scheduling framework to evaluate the potential benefits of charging stations deployed at park-and-ride systems (PR) [13]. Such facilities are gaining popularity in large metropolitans as they enable commuters heading to city centers park their vehicles and continue with a public transport option such as a bus, train, or metro system. Policy-makers use such facilities to reduce traffic congestion, lower emissions, and promote public transportation. Extensive analyses of positive effects and methods for incorporating such systems into existing infrastructure can be found in [14] and [15]. From the known behavior of metro passengers [16], it can be expected that at such parking lots, cars would commonly spend 8-10 hours which makes them ideal for becoming Level 2 chargers for EVs [17]. Moreover, since the presence of such parking lots increase [18] the ongoing projects can easily include such chargers.

The scheduling problem described above is solved through optimization of a combinatorial problem. To do so, the upper 
bound of the potential benefits is calculated for a setting where arrivals and the level of charge of all the vehicles are known in advance. This is done by developing an integer linear problem (ILP) to model the system of interest and the proposed model is evaluated by using real-world data. This is done by first maximizing the potential of flattening the duck curve, and secondly by maximizing the quality of service through the increase in the total amount of charge received by the EVs.

The reminder of this paper is organized as follows. In the next section, the mathematical model used to evaluate the potential of the proposed concept is presented. In Section III details about the data used and the method for generating problem instances. In the next section, the results of the performed computational experiments are presented and analyzed. The paper is finalized with concluding remarks.

\section{Mathematical Model}

In this section, an overview of the proposed mathematical model used to flatten the duck curve is presented. In the proposed model the following assumptions are made. First, the scheduling is done for a time window $\mathcal{T}$ which is divided into a set of periods $\{1, \ldots, T\}$. For each time period $t=1 \ldots T$ a parameter $q_{t}$ is defined corresponding to the base consumption minus the solar generation. The EV charging station will contain chargers of specific rate $s$ (in kilowatts $\mathrm{kW}$ ), and indicates how much a battery can be charged in one time period. In the model, it is assumed that each car $i \in\{1, \ldots, M\}$ visit the charging station and the arrival time $a_{i}$ and departure time $d_{i}$ are known in advance. Each car $i$ has a battery of capacity $f_{i}$ and initial battery charge at arrival $0 \leq b_{i}^{a} \leq f_{i}$. Each battery of an EV can only be charged until its full capacity. At each time period, a maximally allowed charge for the system can be given but in the proposed model it is assumed that it is higher than the total charging potential of the charging station. The flattening of the "duck curve" is done by minimizing the change in total energy need (sum of $q_{t}$ and energy used for charging EVs ) in successive time periods.

In the proposed model, it is further assumed that the activation/deactivation of each charger is controlled. Since the proposed system is modeled using an ILP, let us define binary variables $x_{i t}$, for $i=1, \ldots, M$ and $t=1, \ldots, T$, which state if EV $i$ is charged at time $t$. The objective of the proposed model is to optimize the scheduling of active periods for all the chargers. A minimum charge level is also guaranteed and it is assumed that each EV $i$ must receive a minimal amount of charge $r$ by the time it leaves the station. Similarly, let us define real variables $b_{i t}$, for $\forall i$ and $\forall t$, which indicate the state of the battery (level of charge) of car $i$ at time $t$. In the definition of the ILP, for the sake of simplicity, instead of parameters for arrival $\left(a_{i}\right)$ and departure times $\left(d_{i}\right)$ for a EV $i$, a set of binary parameters $v_{i t}$, for $\forall i$ and $\forall t$, is used to indicate if EV $i$ is at the charging station at time period $t$. Auxiliary variables $h_{t}$, defined for $\forall t$, are used for the total power consumption of the charging station. Moreover, let us define variables $d_{t}$ for $t=1, \ldots, T-1$, which will be used to store the change in energy consumption, or in other words the absolute difference between $h_{t}$ and $h_{t+1}$. Let us first define the constraints in the model as follows.

$$
\begin{array}{rl}
b_{i, 0}=b_{i}^{a} & i=1 \ldots M \\
x_{i t} \leq v_{i t} & i=1 \ldots M, t=1 \ldots T \\
b_{i t+1}=b_{i t}+x_{i t} s & i=1 \ldots M, t=1 \ldots T-1 \\
b_{i t} \leq f_{i} & i=1 \ldots M, t=1 \ldots T \\
b_{i T} \geq b_{i}^{a}+r & i=1 \ldots M
\end{array}
$$

In (1), the constraint sets the initial value of the current battery charge to the state of the battery at arrival. Further, (2) is used to only allow charging at time periods when a vehicle is at the charging station. The constraint related to the change of battery charge of an EV, for one time period is given in (3). The constraints limited to the maximal and minimal level of battery charge of an EV are given in (4) and (5), respectively. The total amount of power used by the charging station is specified in (7). Equations (8)-(11) are used to set the values of variables to $d_{t}$ to the absolute value of the difference between $h_{t}$ of consequent time periods in the standard way. In these equations $M$ is used for a sufficiently large number, and $g_{t}$ are auxiliary values which indicate if $g_{t}>g_{t+1}$ is satisfied. The final constraint (12) is related to finding the maximal difference in power consumption between consecutive time periods.

As previously discussed, for evaluating the model there are two objectives of interest. The first one is minimizing the change in power consumption between consecutive time periods, which corresponds to flattening of the "duck curve". This can be done by minimizing the value of $o$ as in the following equation:

$$
\text { minimize } o
$$

The second one is related to the benefits related to the charging station operator and drivers of EVs. To be more precise, the goal is to maximize the total amount of charge of all the EVs, as in the following equation.

$$
\operatorname{maximize} \sum_{t=1 \ldots T} h_{t}
$$

\section{Generation OF DATA FOR EXPERIMENTS}

To have a better comprehension of the potential benefits of the proposed approach we have generated test instances based on real world data. The group of parameters that needs to be generated is related to $\mathrm{EV}$ visits to the PR facilities. This has been done based on the hourly utilization rate of PR 


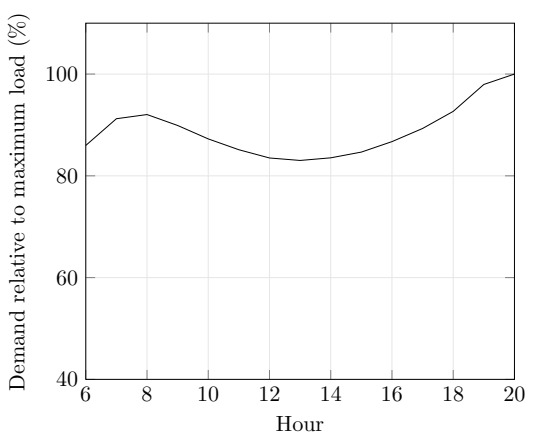

(a) Hourly total demand

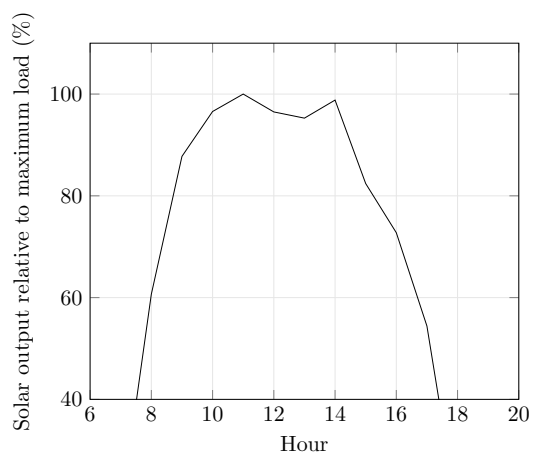

(b) Hourly solar generation

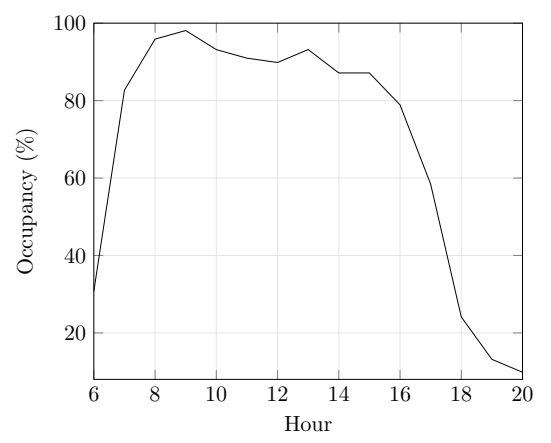

(c) Hourly occupancy of park and ride facility

Fig. 2. Graphical illustration of data used for generating test instances taken from [13]. All the values are given in a normalized form and given in percentage.

facilities taken from [13], a graphical illustration can be seen in Fig. 3(c). In addition, the data from [16] has been used to reflect the behavior of passengers of metro services. To be more precise, this study shows that $70 \%$ of performed trips are related to work and the rest to the other activities. In practice, in the method for generating problem instances $70 \%$ of the EV's parking time lengths are randomly selected from 8 to 10 hours. For other activities the parking time has randomly selected from 2-6 hours. In both cases a uniform distribution has been used. The times of arrivals of individual vehicles has been randomly generated in a way that the hourly occupancy of the PR facility would correspond to the data from [13]. To be more precise, the total number of vehicles at PR facility at each time period has been made to be proportional to the level of occupancy at that time period. The battery types of EVs have been randomly generated based on data related EV sales taken from [19] and corresponding battery sizes [20]. The state of the charge of individual batteries was randomly generated form the range $15 \%$ - $80 \%$ using a uniform distribution.

The generated test instances are used to assess reductions in ramping requirements for varying percentages of solar generation and EV penetration levels. The information on the total demand and production from solar generation are adopted from publicly available data in California [21] and presented in Figs. 3(a) and 3(b). For demand response programs, it has been shown that the additional benefits of scheduling smart appliances for more than a certain number of households does not provide significant benefits [22]. It is reasonable to assume a similar behavior for scheduling the charging of EVs. Because of this, in the method for generating problem instance, the number of vehicles $N$ is initially selected and the maximal total energy use for charging is calculated based on the arrival and departure times for vehicles (generated as previously described) for each time period. Next, these values are used to generate the values of the load and solar generation to satisfy the proportion. In other words, the normalized values given in Fig. 2 are multiplied by constants to get the correct proportion for EV consumption $E$, base load $L$ and solar production $S$.

\section{Results}

In this section, we present the results of the performed computational experiments. The proposed ILP has been implemented using OPL in IBM ILOG CPLEX Optimization Studio Version: 12.6.1.0, and executed using the default solver settings. In generating the problem instances the time period between 6:00 and 20:00 has been used to mimic actual operational times of parking lots. Each time period in the model corresponded to 15 minutes, which means the value of parameter $T$ is 56 . In the generated problem instances a fixed number $N=500$ is used for the number of EVs visiting the PR facility. The potential of scheduling EV charging to minimize issue related to duck curve are evaluated for settings where $10 \%, 20 \%$ and $30 \%$ of the maximal load is produced from solar generation. The assumed power of the chargers is 4 $\mathrm{kW}$, which translates to $s=1$ inside of the model. The chosen minimal amount of charge received by an EV was equivalent to an hour of charging, which inside the model translates to $r=4$. The maximal amount of energy used for EV charging was $2.5 \%, 5.0 \%$ and $10.0 \%$ of the total energy consumption. Two objective given in Eqs. (13), (14) have been used in the following way. First the model having constraints given in Eqs. (1)- (12) is used to find the value (Min) of minimal change in power consumption $o$ is found. Next, in the constraint given in (12) $o$ is substituted with Min and the model is solved for objective function given in (14), related to the total energy used for charging the EVs. The focus of the paper is on the potential benefits of scheduling EV charging and not on the solution method for the model.On the other hand, it should be noted that the time needed to find an optimal solution for a problem instance would be less than a second.

The level of flattening of the duck curve, based on the proposed model, by optimizing the charging periods of EVs at PR facilities can be seen in Fig. 3. Several things can be observed. In case only $2.5 \%$ energy used for EV charging the positive effect is relatively small but noticeable. When the energy use for the same purpose is relatively high (10\%) and the positive effect becomes significant. When $10 \%$ of electric power comes from solar generation the issue of the duck curve is to a large extend resolved. In case of higher 


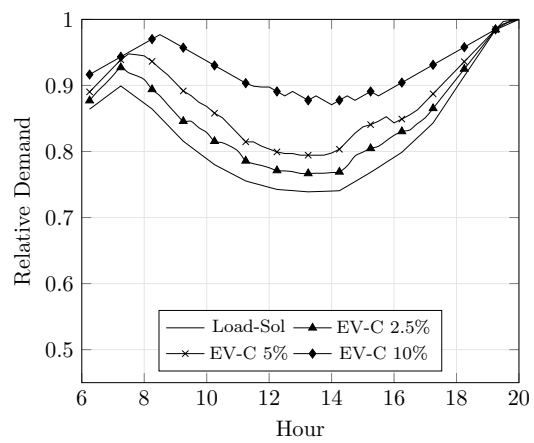

(a) Solar generation $10 \%$
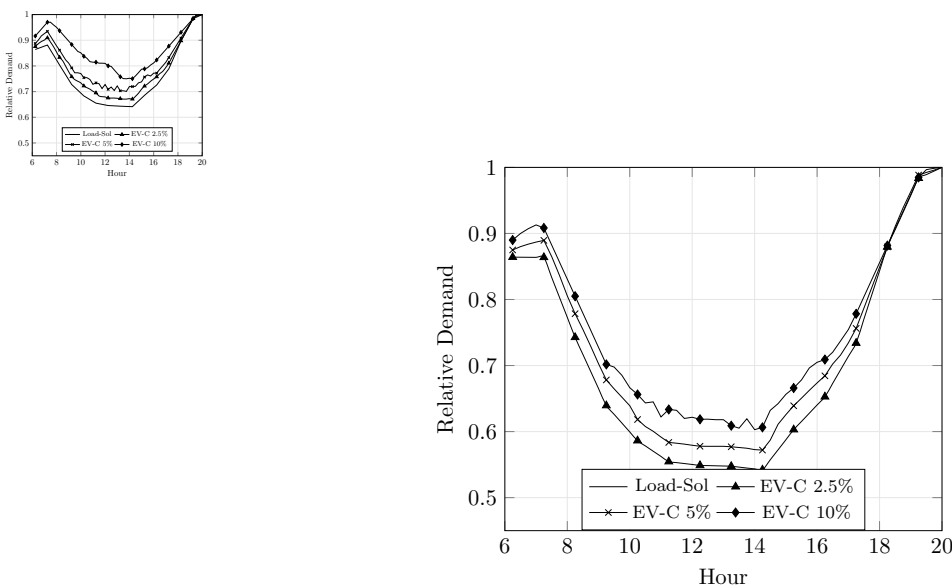

(b) Solar generation $20 \%$

(c) Solar generation $30 \%$

Fig. 3. Comparison of the normalized values for required generation of electricity for different levels of solar production with different levels of consumption related to charging of EVs. The notation Load - Sole is used for the case there is not EV charging, and $E V-C$ to indicate the part of the load that is related to EV charging.

TABLE I

Percentage (\%) OF Reductions IN RAMPING ReQUiREMENTS

\begin{tabular}{llll}
\hline & EV 2.5\% & EV 5\% & EV 10\% \\
\hline Solar 10\% & 3.76 & 7.51 & 17.85 \\
Solar 20\% & 4.60 & 9.26 & 16.93 \\
Solar 30\% & 5.52 & 11.22 & 24.61 \\
\hline
\end{tabular}

amount of power generated from the same type of source, the negative effects are lowered but still significant. An unexpected effect of this type of scheduling is a significant increase at the morning peak demand but with out increasing the change in energy use between time periods. This is a consequence that the highest occupancy of the PR facility is at this time. Another observation that can be made is that although the use of the proposed scheduling scheme results in a lower maximal change in energy need between successive time periods, there is a lower number of them when the energy need is relatively constant.

Effectiveness of the proposed model can also be quantified based on the amount of eliminated ramping requirements. For instance, the difference between minimum and maximum load for $30 \%$ solar is $45 \%$ of the total consumption from $2: 25 \mathrm{pm}$ to 8:00 pm (EV-C 10\% case). However, by optimally scheduling EVs (e.g., EV- C 10\%), this requirement reduces to $32 \%$ ramping in the same amount of time. In Table I, we present percentage of reductions in ramping requirements compared to no EV scheduling case. It can be easily observed that the proposed method is very effective in flattening load curves.

\section{Conclusion}

Duck curves, introduced by high penetration of renewables, creates new operational constraints and increases power system operational cost. In this paper, we have presented an analysis of the potential benefits of scheduling charging periods of Level $2 \mathrm{EV}$ chargers at park-and-ride facilities to flatten duck curves. To achieve this, we have introduced a new mathematical model using integer linear programming. The evaluation has been done based on real-world data on solar generation, hourly demand, driver behavior and EV sales. The model and data have been used to asses the impact of such systems for difference levels of adoption of EVs and expansion of solar generation. The performed computational results have shown significant savings can be achieve by optimally charging stationary EVs

In the future, we plan to extend this research in two main directions. The first one is observing the problem form a multi-objective perspective to be able to have a more in depth understanding. The second direction is in extending the proposed model to an online version where only partial information about EVs (battery state, arrival and departure times) are known.

\section{REFERENCES}

[1] M. Obi and R. Bass, "Trends and challenges of grid-connected photovoltaic systems-a review," Renewable and Sustainable Energy Reviews, vol. 58, pp. 1082-1094, 2016.

[2] P. Denholm, M. O'Connell, G. Brinkman, and J. Jorgenson, "Overgeneration from solar energy in california. a field guide to the duck chart," National Renewable Energy Lab.(NREL), Golden, CO (United States), Tech. Rep., 2015.

[3] M. A. Zehir, A. Batman, and M. Bagriyanik, "Review and comparison of demand response options for more effective use of renewable energy at consumer level," Renewable and Sustainable Energy Reviews, vol. 56, pp. 631-642, 2016.

[4] D. Meyer and J. Wang, "Integrating ultra-fast charging stations within the power grids of smart cities: a review," IET Smart Grid, vol. 1, no. 1, pp. 3-10, 2018. 
[5] I. Rahman, P. M. Vasant, B. S. M. Singh, M. Abdullah-Al-Wadud, and N. Adnan, "Review of recent trends in optimization techniques for plugin hybrid, and electric vehicle charging infrastructures," Renewable and Sustainable Energy Reviews, vol. 58, pp. 1039-1047, 2016.

[6] X. Wu, "Role of workplace charging opportunities on adoption of plugin electric vehicles-analysis based on gps-based longitudinal travel data," Energy Policy, vol. 114, pp. 367-379, 2018.

[7] B. Ferguson, V. Nagaraj, E. C. Kara, and M. Alizadeh, "Optimal planning of workplace electric vehicle charging infrastructure with smart charging opportunities," in 2018 21st International Conference on Intelligent Transportation Systems (ITSC). IEEE, 2018, pp. 11491154.

[8] R. S. Levinson and T. H. West, "Impact of convenient away-from-home charging infrastructure," Transportation Research Part D: Transport and Environment, vol. 65, pp. 288-299, 2018.

[9] P. J. Tulpule, V. Marano, S. Yurkovich, and G. Rizzoni, "Economic and environmental impacts of a pv powered workplace parking garage charging station," Applied Energy, vol. 108, pp. 323-332, 2013.

[10] Y. Zhang and L. Cai, "Dynamic charging scheduling for ev parking lots with photovoltaic power system," IEEE Access, vol. 6, pp. 56995$57005,2018$.

[11] R. Dhawan and S. Prabhakar Karthikeyan, "An efficient ev fleet management for charging at workplace using solar energy," in 2018 National Power Engineering Conference (NPEC), March 2018, pp. 1-5.

[12] Z. Wei, Y. Li, Y. Zhang, and L. Cai, "Intelligent parking garage ev charging scheduling considering battery charging characteristic," IEEE Transactions on Industrial Electronics, vol. 65, no. 3, pp. 2806-2816, March 2018.

[13] L. K Cherrington, J. Brooks, J. Cardenas, Z. Elgart, L. David Galicia T. Hansen, K. Miller, M. J. Walk, P. Ryus, C. Semler, and K. Coffel, "Decision-making toolbox to plan and manage park-and-ride facilities for public transportation: Guidebook on planning and managing parkand-ride," Tech. Rep., 012017.

[14] T. Zijlstra, T. Vanoutrive, and A. Verhetsel, "A meta-analysis of the effectiveness of park-and-ride facilities," European Journal of Transport and Infrastructure Research, vol. 15, no. 4, 2015.

[15] L. Cornejo, S. Perez, R. L. Cheu, and S. Hernandez, "An approach to comprehensively evaluate potential park and ride facilities," International Journal of Transportation Science and Technology, vol. 3, no. 1, pp. 1-18, 2014.

[16] J. Neff, "A profile of public transportation passenger demographics and travel characteristics reported in on-board surveys," American Public Transportation Association and others, 2007.

[17] M. Yilmaz and P. T. Krein, "Review of battery charger topologies, charging power levels, and infrastructure for plug-in electric and hybrid vehicles," IEEE transactions on Power Electronics, vol. 28, no. 5, pp. 2151-2169, 2013.

[18] J.-M. Ruan, B. Liu, H. Wei, Y. Qu, N. Zhu, and X. Zhou, "How many and where to locate parking lots? a space-time accessibility-maximization modeling framework for special event traffic management," Urban Rail Transit, vol. 2, no. 2, pp. 59-70, Jun 2016. [Online]. Available: https://doi.org/10.1007/s40864-016-0038-9

[19] InsideEVs. (2019) Monthly plug-in ev sales scorecard. [Online]. Available: https://insideevs.com/monthly-plug-in-sales-scorecard/

[20] Wikipedia. (2019) Electric vehicle battery. Accessed 2019-04-04 [Online]. Available: https://en.wikipedia.org

[21] California ISO. (2019) California ISO. [Online]. Available: http://www.caiso.com

[22] R. Jovanovic, A. Bousselham, and I. Bayram, "Residential demand response scheduling with consideration of consumer preferences," Applied Sciences, vol. 6, no. 1, p. 16, 2016. 